\section{Rubella Policies for Hospitals and Health Workers}

Stephen C. Schoenbaum, M.D., M.P.H.

There have been several recent articles and editorials on the problems of nosocomial rubella and rubella immunization of hospital workers. ${ }^{1-4}$ To understand any discussion of the problem of rubella in hospitals, one must be cognizant of a few basic considerations:

- Despite the great decline in the occurrence of rubella in children which followed the licensure of rubella vaccine in 1969 , the reported occurrence of rubella in young adults, ages $15-25$, is approximately the same now as it was a decade ago;

- Compared to the general population, hospital workers are disproportionately likely to be women and of childbearing age. Between 10 and 20 percent of childbearing age women remain susceptible to rubella despite a decade of use of the rubella vaccine; and

- Pregnant women are often dependent on hospitalbased services, and it is quite possible that a single infected hospital employee could transmit rubella to several susceptible pregnant patients (some instances of transmission have been documented).

With these considerations in mind it has been argued that hospitals should detect and/or immunize their susceptible staff; and the Public Health Service Immunization Practices Advisory Committee has recommended, "To protect susceptible female patients and female employees, persons (both male and female) working in hospitals and clinics who might contract rubella from infected patients or who, if infected, might transmit rubella to pregnant patients should be vaccinated against rubella, unless there are contraindications."

Each hospital must face the task of adopting, instituting, and maintaining a consistent policy on rubella. It is not a simple procedure; and as with all other policy decisions, the benefits, costs and risks must be weighed. There are several strategies to be evaluated, including: (A) do nothing; (B) have a voluntary program for screening staff and employees of the hospital for susceptibility to rubella and immunize susceptibles voluntarily; (C) have a mandatory program of screening for all hospital staff and employees and a voluntary or mandatory immunization program for susceptibles; and (D) have a voluntary or mandatory immunization program without pre-screening. Variations might include having one type of policy for those persons in the hospital most likely to care for pregnant patients with rubella (the obstetric and pediatric areas, ultrasound and other selected laboratories, etc.) and another policy for the remainder of hospital employees and staff.

An ostrich-like policy of doing nothing is unattractive for several reasons. While cheap and superficially appealing, it leaves the hospital open to chaos should one or more of its employees develop rubella. In such a circumstance no one really knows who is susceptible, and a crash program must ensue. A hospital adopting this policy could be lucky and never have to face the problem of rubella amongst its employees. However, as long as rubella remains prevalent in the young adult population, the "do nothing" hospital will have to be extremely lucky to avoid having the problem at some time.

The strategies of voluntary screening or voluntary immunization without screening are also unattractive. When a hospital with this type of policy faces the actual problem of rubella among its employees, there will be a large amount of missing information on the immune status of its employees; and again a crash program will be necessary.

Thus, the potential for rubella actually occurring within the hospital necessitates a mandatory approach. For all practical purposes, it comes down to a choice between mandatory screening and/or mandatory immunization. Mandatory screening programs carry only the risk of venipuncture. Any hospital large enough to perform its own serologic testing for rubella faces only the actual costs for these tests and not the charges that might be billed by an outside laboratory. If the tests are run simply to detect the presence of antibody (a single screening dilution of the serum), the costs to the hospital (cont'd on p. 116$)$ 
(cont'd from p. 366)

should be quite low - probably less than the cost of a dose of rubella vaccine. It should also be easier to cajole reluctant employees into having a simple screening test than into receiving an immunization that they really don't want. Since less than one in five employees will turn out to be susceptible, relatively few will have to be reapproached for immunization. In addition, a hospital should be able to exclude susceptibles who refused immunization from working or from patient contact during known rubella epidemics. While this could potentially lead to exclusion of $15-20$ percent of the hospital's staff, it is likely that the hospital would exclude only employees working in certain areas and could make up the deficit with immune staff borrowed from other areas. All of these considerations suggest that mandatory screening without mandatory immunization of existing employees is a reasonable, though not necessarily optimal, strategy.

Institution of a mandatory screening and immunization program for new employees has proven to be a relatively simple policy to institute and maintain. Immunity to rubella can be made a condition of employment. The policy can be administered through the employee health service and incorporated into the routine medical procedures for new employees.

If a hospital were to opt for a policy of mandatory immunization of existing employees, it should consider giving the vaccine to all employees without prescreening. A serum specimen could be obtained from female employees and staff at the time of vaccination and simply set aside. If it subsequently appeared that a woman had been vaccinated inadvertently in the first trimester of pregnancy, her serum sample could be retrieved and tested. There would be no risk if she was already immune; and, indeed, the risk of inadvertent rubella immunization of susceptible pregnant women now appears to be so low that it should not be considered a routine indication for interruption of pregnancy.

The selection of a policy of mandatory immunization without prescreening depends, in part, upon the relative costs of the vaccine and the screening program to the hospital. It is also dependent upon the logistics of the hospital and how difficult it may be to reach some employees twice, once for screening and again for immunization. The risks should be about the same whether one gives vaccine to all employees or just to proven susceptibles - persons who are immune to rubella have virtually no reactions to the vaccine, the reactions being almost totally attributable to the live virus infection which occurs in the susceptibles. It can prove difficult to convince adults to accept a vaccine for a disease to which they believe they are immune. Since histories of rubella and undocumented histories of immunization are notoriously unreliable, however, only the presence of antibody or documentation of prior vaccination should be considered evidence of immunity and a basis for nonparticipation in a mandatory immunization program.

The legal basis for mandatory immunization of existing employees appears to be related to the hospital's obligation to protect the health and safety of its patients including the unborn offspring of pregnant women. I am not aware of any legal challenges to a hospital's insisting on mandatory immunization of existing employees; but it is possible that hospitals that nominally have adopted such a policy have not pressed their most intransigent holdouts. Another legal issue related to mandatory immunization is liability for vaccine reactions, most probably arthritis. On the whole, reactions to vaccination will not be serious or disruptive of normal work schedules. ${ }^{1,2}$ Should a serious reaction occur, the hospital could probably handle it under Workmen's Compensation provisions, in which case the hospital's liability is limited. The employee could probably challenge this approach, though to-date I am unaware of such a challenge.

It must be remembered that the persons who work in hospitals and who could expose susceptible parties or visitors are not limited to those who receive a direct hospital salary. Physicians and students must be involved in any program. In practice, this has been a major problem: In at least two instances ${ }^{1,2}$ physicians have been considerably less likely than other members of the hospital staff to participate in the program. It is very difficult for the overall program to be credible or effective when physicians are not willing to cooperate with it.

Achieving control over non-salaried staff requires drastic measures, but is not impossible to accomplish. In 1979, rubella occurred among employees of several Boston hospitals and in one there was a common-source outbreak. 'The Lying-In Division of the Boston Hospital for Women (an obstetrical service now part of the Brigham and Women's Hospital) had five cases of rubella among its 1100 employees. In addition to requiring proof of immunity from its salaried staff it was decided that all students should be included in the program. As a result, immunity.to rubella has become a pre-requisite for all student groups by agreement with their parent institutions. Physicians were threatened with loss of privileges if they did not supply evidence of immunity. Nineteen of 131 physicians were found to be susceptible. All have complied with the program. In this large obstetrical hospital it also was possible to obtain rubella titers on over 94 percent of all employees with persistent efforts over a three month period of time; and all of the 65 persons who were missed were casual or weekend employees. Of those who were tested, 15 percent were susceptible ( 163 persons) and 96 percent of the susceptibles (all but 7) were immunized. Incidentally, when the Boston Hospital for Women merged physically with the Peter Bent Brigham and Robert Breck Brigham Hospitals in 1980 it was decided to have a policy of mandatory screening for all new employees in the combined Brigham and Women's Hospital and mandatory immunization for all workers with patient contact.

Finally, it should be obvious that nothing about the foregoing discussion of policies and strategies is specific to hospitals. The issues apply equally to all health care workers in non-hospital settings with similar possibilities of exposure to rubella and exposing patients. Moreover, the real threat of rubella is to susceptible pregnant women; and the best way to avoid the problem of rubella syndrome is to ensure than no women who become pregnant are susceptible. Control of rubella syndrome cannot be 
achieved simply by immunizing hospital or health workers. The pregnant woman may still be exposed to rubella in her workplace, in public, or even at home. It is to be hoped that the current emphasis on school immunization for communicable diseases including rubella, and the increased attention that the Public Health Service Immunization Practices Advisory Committee has been trying to bring to vaccination of all susceptible young women' will lead to the ultimate eradication of congenital rubella syndrome.

ACKNOIVLEDCENENT: I would like to thank Barbara Leece, R.N. and Ruth Brown for the information on the results for the rubella program at the Boston Hospital for Women and B. Frank Polk, M.D. for revieuing the manuscript.

Stephen C. Schoenbaum, M.D., M.P.H. Hariard Community Health Plan One Fenu'ay Plaza Boston, MA 02215 (Address reprint requests to Dr. Schoenbaum)

\section{REFERENCES}

1. Polk BF, White JA, DeGirolami PC. Modlin JF: An outbreak of rubella among hospital personnel. N Engl J Med. 1980; 303:541545.

2. Orenstein WA, Heseltine PNR, LeGagnoux SJ, Portnoy B: Rubella vaccine and susceptible hospital employees: poor physician participation. JAMA. 1981; 245:711-713.

3. Nosoromial rubella infection - North Dakota, Alabama, Ohio. Morbid Mortal Weekly Rep. 1981; 29:629-631.

4. Preblud SR, Hinman AR: Rubella vaccination of hospital employees, an editorial. JAMA. 1981; 245:736-737.

5. Rerommendation of the Immunization Practices Advisory Committee (ACIP): Rubella prevention. Morbid Mortal Weekly Rep. 1981: 30:37-42,47. 